\title{
Characterizing Working Catalysts with Correlated Electron and Photon Probes
}

Eric A. Stach, ${ }^{1}$ Yuanyuan Li, ${ }^{2}$ Shen Zhao, ${ }^{3}$ Andrew Gamalski, ${ }^{1}$ Dmitri Zakharov, ${ }^{1}$ Ryan Tappero, ${ }^{4}$ Karen Chen-Weigart, ${ }^{4}$ Juergen Thieme, ${ }^{4}$ Ulrich Jung, ${ }^{3}$ Anika Elsen, ${ }^{3}$ Qiyuan Wu, ${ }^{5}$ Alexander Orlov, Jingguang Chen, ${ }^{6}$ Ralph G. Nuzzo ${ }^{3}$ and Anatoly Frenkel ${ }^{2}$

1. Center for Functional Nanomaterials, Brookhaven National Laboratory, New York, NY 11973

2. Department of Physics, Yeshiva University, New York, NY 10016

3. Department of Chemistry, University of Illinois at Urbana-Champaign, Illinois 61820

4. National Synchrotron Light Source II, Brookhaven National Laboratory, New York, NY 11973

5. Materials Science and Engineering Department, Stony Brook University, Stony Brook 11794

6. Department of Chemical Engineering, Columbia University, New York, NY 10027

Heterogeneous catalysts often undergo dramatic changes in their structure as the mediate a chemical reaction. Multiple experimental approaches have been developed to understand these changes, but each has its particular limitations. Electron microscopy can provide analytical characterization with exquisite spatial resolution, but generally requires that the sample be imaged both ex situ and ex post facto. Photon probes have superior depth penetration and thus can be used to characterize samples in operando (i.e. when they are actively working). But they generally lack spatial resolution and thus give only ensemble average information.

We have taken advantage of the recent developments in closed-cell microscopy methods ${ }^{1}$ to develop an approach that allows us to successfully combine electron, x-ray and optical probes to characterize supported nanoparticle catalysts in operando. By measuring the reaction products at each stage of the reaction, we can directly correlate the information that can be obtained from each approach, and thus gain a deep insight into the structural dynamics of the system.

We will describe how we can use this approach to correlate x-ray absorption spectroscopy (both nearedge and extended fine structure), scanning transmission electron microscopy and infrared microspectroscopy to understand how $\mathrm{Pt}$ and $\mathrm{Pd}$ nanoparticles supported on silica undergo structural changes during the room temperature hydrogenation of the ethylene, and how we can direct measure and describe the reaction products on the surfaces of the nanoparticles as the reaction proceeds. By combining these approaches, we can track the interplay between nanoparticle reduction, coarsening, and the specific surface species at different stages of the reaction. ${ }^{2}$ (Figure 1) We will also show how this approach can be used to understand the partitioning that occurs in bimetallic nanoparticles during oxidation and reduction at elevated temperatures, with a focus on the NiPt system.

The presentation will focus on the development and application of experimental methods, including the high temperature atmospheric pressure electron microscopy, the direct measurement of reaction products using gas chromatography-mass spectrometry and the ability of a newly developed electron microscope for operando microscopy (based on the FEI Talos platform) to characterize bimetallic nanoparticles through energy dispersive $\mathrm{x}$-ray spectroscopy [1-3].

References:

[1] de Jonge, N. and Ross, F.M., Nat. Nano., 6 (2011), p. 695. 
[2] Li, Y. et al, The Complex Structural Dynamics of Catalysts as Revealed by Correlated Imaging and Spectroscopy Probes, submitted.

[3] This research project was supported, in part, by DOE BES under Contract No. DE-FG02-

03ER15476. Development of the micro-cell approach was supported, in part, by an LDRD grant by Brookhaven National Laboratory. Research was carried out at the Center for Functional Nanomaterials and the National Synchrotron Light Sources I and II at the Brookhaven National Laboratory, which is supported by the U.S. Department of Energy, Office of Basic Energy Sciences, under Contract No. DEAC02-98CH10886 and DE-SC0012704 .
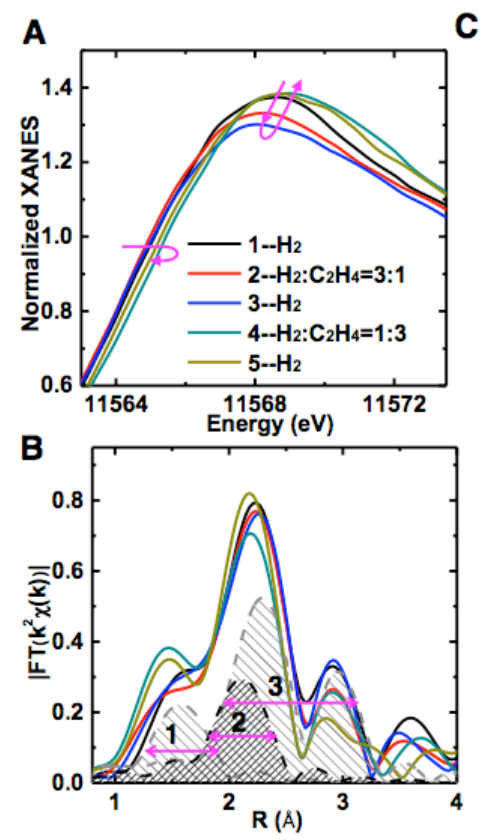

C
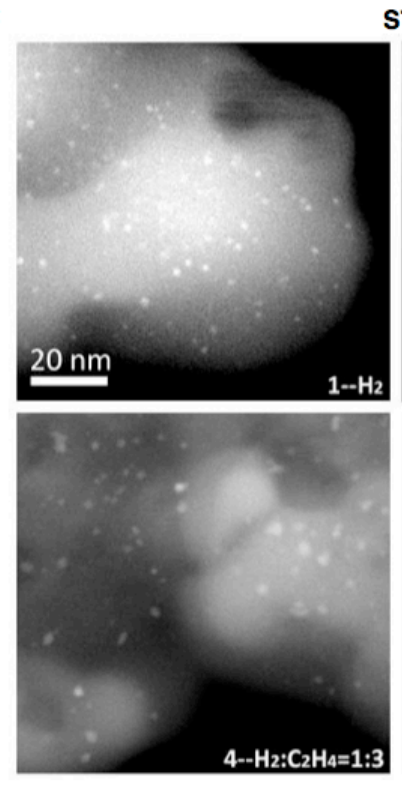

STEM images at pressure: 1 atm.
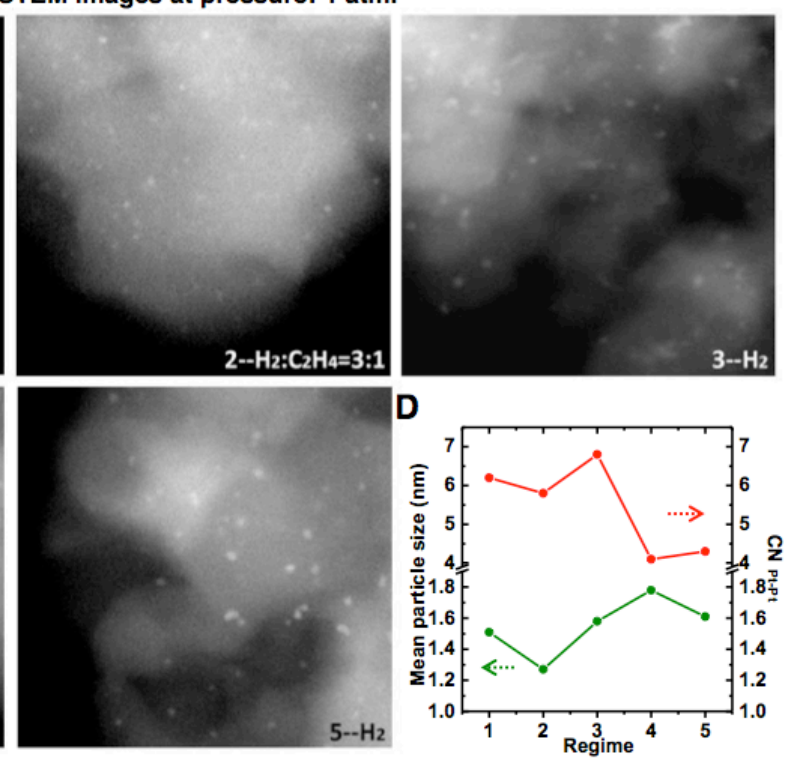

Figure 1. Experimental results from operando XAFS and STEM. (A) X-ray absorption near edge spectroscopy, (B) Fourier transform magnitudes of $\mathrm{Pt}_{2}$ edge EXAFS spectra, and (C) STEM images, all measured in operando, during different reaction regimes. EXAFS and STEM results are displayed in (D) for all regimes. The green line indicates the mean particle sizes, as obtained by the STEM image analysis. The red line shows the change of Pt-Pt coordination number obtained from XAFS analysis. 